\title{
Dynamics of an Optically Injected Diode Laser Subject to Periodic Perturbation: Occurrence of a Large Number of Attractors, Bistability and Metastable Chaos
}

\author{
Thiery Tchinda*, Zeric Njitacke, Theophile Fozin Fonzin, Hilaire Fotsin \\ Department of Physics, Faculty of Sciences, University of Dschang, Dschang, Cameroon \\ Email address: \\ thierytchinda@yahoo.fr (T. Tchinda),njitackezeric@gmail.com (Z. Njitacke), fozintheo@gmail.com (T. F. Fonzin), \\ hbfotsins@yahool.com (H. Fotsin) \\ ${ }^{*}$ Corresponding author
}

\section{To cite this article:}

Thiery Tchinda, Zeric Njitacke, Theophile Fozin Fonzin, Hilaire Fotsin. Dynamics of an Optically Injected Diode Laser Subject to Periodic Perturbation: Occurrence of a Large Number of Attractors, Bistability and Metastable Chaos. Science Journal of Circuits, Systems and Signal Processing. Vol. 8, No. 2, 2019, pp. 66-75. doi: 10.11648/j.cssp.20190802.15

Received: November 14, 2019; Accepted: December 11, 2019; Published: December 25, 2019

\begin{abstract}
It is well known that during a certain interval of time, a molecule of gas in the beam path from the pump laser (master) to the injected laser (slave) can decrease the power injected [1]. In this contribution, we consider a well-known rate equation model of semiconductor laser to explore numerically the effect of a periodic perturbation from the pump laser (master) on the dynamical behavior of the injected laser (slave). Using nonlinear diagnostic tools such bifurcation diagrams, graph of maximum Lyapunov exponent, phase portraits, Poincare sections, basin of attraction and two parameter diagrams, the dynamical behavior of the model is analyzed in terms of its parameters. The coexistence of periodic and chaotic attractors as well as the occurrence of ten different attractors (symmetric and asymmetric) and transient chaos are demonstrated. Finally, PSpice simulations are performed to support numerical results.
\end{abstract}

Keywords: Laser Model, Occurrence of Attractors, Bifurcation Analysis, Bistability Metastable, Circuit Emulator

\section{Introduction}

With help of some dynamical analysis tools, several phenomena including physical, social and economic have been modeled. Among these models, we have neuron model [2], financial model [3], atmospheric model [4], satellite model [5] and laser model [6] just to name few. One can note that, the models subject to periodic excitation have received a lot of investigation. Murali et al., 1991[7] reported complex phenomena in Chua's circuit subject to external sinusoidal signal. He revealed several phenomena in this circuit including period doubling, quasi periodicity, intermittency and coexistence of multiple attractors. Amanda et al., 2012 [8] showed that Rossler model under influence of external sinusoidal force may able to generate hyper chaos and both chaos-order. [9] exploited two-neuron based non-autonomous memristive Hopfield neural network to investigate dynamical behaviors including bursting. Recently, Ngo Mouela et al., 2019 [10] exploited a simple nonautonomous Jerk system with generalized nonlinearity to investigated rich dynamical behaviors including hyperchaos, chaos, intermittency and coexistence of multiple attractors. In the same research way, semiconductor lasers with optical injection have received a lot of investigation. Harder et al., 1982 [11] realized the noise equivalent circuit of a semiconductor laser diode which is derived from the rate equations subject to Langevin noise. Hwang et al., 1999 [12] generated chaos by intrinsic spontaneous-emission noise in an optically injected semiconductor laser. Garbin et al., 2017 [13] study experimentally an excitable semiconductor laser optically injected in neuron like excitable regime. He underlined the existence of a refractory period during which perturbations are not able to elicit an excitable response. Gustave et al., 2016 [14] analyzed the stability of stationary solutions in an optically injected semiconductor laser. Pimenov et al., 2014 [15] investigated the effect of coherent single frequency injection on two-section semiconductor lasers. He reported some phenomena including bistability and hysteresis. Pramod et al., 2013 [16] reported that, if the slave laser provides sufficient frequency filtering the, selective injection locking of 
a single-frequency may also be achieved on a multi-mode semiconductor laser injected by a multi-frequency seeding beam. Wieczorek et al., 2005 [17] discovered a new dynamical effect (multipulse excitability) and some phenomena including cascades of bifurcation, multistability and sudden chaotic transitions in an optically injected semiconductor laser. Recently, Torre et al., 2019 [1] explored the combined effect of excitability and extreme pulse emission for the detection of variation in the strength of the injected field of semiconductor laser. It is important to note that these different investigations have been carry out on the dynamics of semiconductor lasers because of their potential applications in engineering and particularly in injection locking [18] frequency stabilization [19] linewidth narrowing and chirp reduction [20]. In order to characterize the impact of a periodic perturbation from the pump laser (master) on the dynamical behaviors of the injected laser (slave), the paper is organized as follows. In section 2, we describe the proposed model of optically injected diode laser. In section 3, various bifurcation diagrams are plotted as injected field strength (resp amplitude of external perturbation and excitation frequency) are varied. Additionally, phase portraits, Poincare sections, two parameters diagrams and cross section basin of initial condition are plotted in order to underlined the occurrence of ten distinct attractors and the coexistence of periodic and chaotic attractors. To confirm theorical result, some PSpice simulations are investigated in section 4 and the result obtained agree with theoretical ones. Finally, in section 5 we summarize our work.

\section{Methods}

\subsection{Model Description}

It is well known that a single mode semiconductor laser optically driven by monochromatic light is given by the following rate equation:

$$
\left\{\begin{array}{c}
\dot{E}=\gamma+\left(\frac{1}{2}(1+i \alpha) n-i \omega\right) E \\
\dot{n}=-2 \Gamma n-(1+2 B n)\left(|E|^{2}-1\right)
\end{array}\right.
$$

Where $B$ and $\Gamma$ are the material properties of the laser, $\alpha$ the linewidth enhancement factor, $\omega$ the laser frequency (detuning), $E_{x}$ and $E_{y}$ the real and imaginary part of the complex electric field respectively, $n$ the normalized population inversion, $\gamma$ the injected field strength. Considering the effect of an optical perturbation [1] due to the presence during a certain time interval of light ray in the beam path from the pump laser (master) to the injected laser (slave), the mathematical model of the semiconductor laser subject to periodic optical perturbation is given as:

$$
\left\{\begin{array}{c}
\dot{E}_{x}=\gamma+\frac{1}{2} E_{x} n+\left(\omega-\frac{1}{2} \alpha n\right) E_{y}-F_{0} \sin (2 \pi f t) \\
\dot{E}_{y}=-\left(\omega-\frac{1}{2} \alpha n\right) E_{x}+\frac{1}{2} E_{y} n \\
\dot{n}=-2 \Gamma n-(1+2 B n)\left(E_{x}^{2}+E_{y}^{2}-1\right)
\end{array}\right.
$$

In this model, $F_{0}$ is the external amplitude and $f$ the external frequency.

\subsection{Numerical Methods}

In this section, we explore the dynamics of an optically injected diode laser subject to external perturbation. Using common nonlinear dynamical tools such as bifurcation diagrams, Lyapunov exponent Poincare section, two parameter diagrams, cross section of initial condition and phase portraits. Computation are performed in FORTRAN (with variables and parameters in double precision mode). The system was simulated for a sufficiently long time and transient was always discarded (except when analyzing metastable dynamics).

\section{Results}

\subsection{Bifurcation Analysis}

In this subsection, using bifurcation diagrams as argument, we investigate the effect of external amplitude (resp excitation frequency) on the dynamic of a laser model. We plotted in Figure 1 bifurcation diagrams and the corresponding graph of Lyapunov exponents when excitation frequency varies from 0 to 2.2. The bifurcation diagrams show dynamical behaviors including chaos, torus and periodic oscillations. According to Figure 1, excitation frequency is fixed at 1 and we plotted as shown in Figure 2 bifurcation diagram and corresponding graph of Lyapunov exponent when external amplitude varies in the range $0 \leq F_{0} \leq 5$. In Figure 2, it is easy to observe dynamical behaviors including periodic, quasi-periodic oscillations and chaotic behaviors when the control parameter $F_{0}$ is monitored. One can note that in the absence of external excitation (ie $F_{0}=0$ ), the dynamic of laser is chaotic. Whereas dynamic is periodic (resp torus) when $F_{0} \in[2.85 ; 3.25]$ (resp $\left.F_{0} \in[4.11 ; 4.89]\right)$. Using the same range of parameters in Figure 2 we plotted two bifurcation diagrams and corresponding graph of Lyapunov exponent when laser injection pump varies between 0 and 1 . The diagrams of Figure $3 a$ (resp Figure $3 b$ ) were plotted for two discrete value of external amplitude. One can easily note that when external amplitude is fixed at 0 (resp 3 and 5), the laser described different dynamical behaviors including periodic, quasi-periodic oscillations, torus and chaotic dynamics. In Figs $3 a$ and $3 b$, the abrupt variation from chaotic to periodic motion observed at 0.6 (resp 0.94) see (resp Figure 3a and Figure 3b) highlight the phenomena of saddle node bifurcation (SNB). Figure 3 reveal that for the low pump value and in absence of external perturbation, the laser performs periodic behaviors. For the same range of parameters and for $\gamma=0.04$ (low value), some sample numerical phase portraits and corresponding Poincare sections were plotted to confirm different scenario observed in Figure 2 (see Figure 4). In Figure 4, one can note the occurrence of symmetric attractors (Figure. $4 \mathrm{~g}, \mathrm{~h}, \mathrm{~d}$ ) and asymmetric ones (Figure 4a, b, c, e, f, j). 


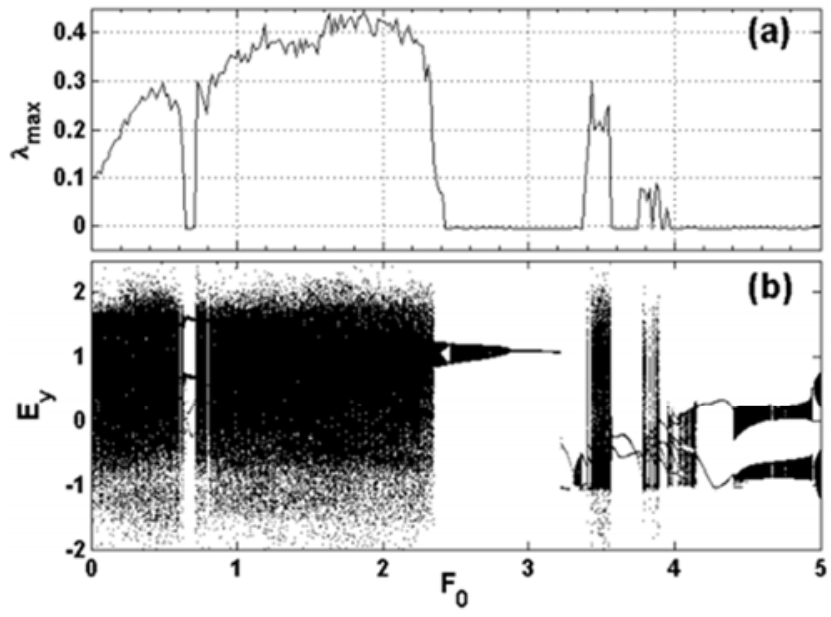

Figure 1. (a) Bifurcation diagrams showing local maxima of the imaginary part of the complex electric field $E_{y}$ and corresponding graph of Lyapunov exponents (b) versus external frequency $\omega_{1}$.
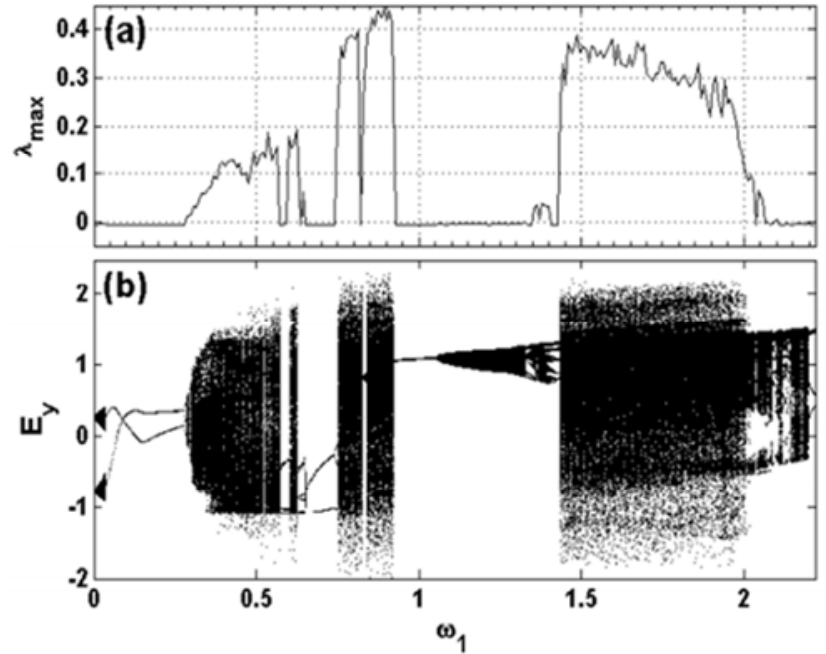

Figure 2. (a) Bifurcation diagrams showing local maxima of the imaginary part of the complex electric field $E_{y}$ and corresponding graph of Lyapunov exponents (b) versus external amplitude $F_{0}$.
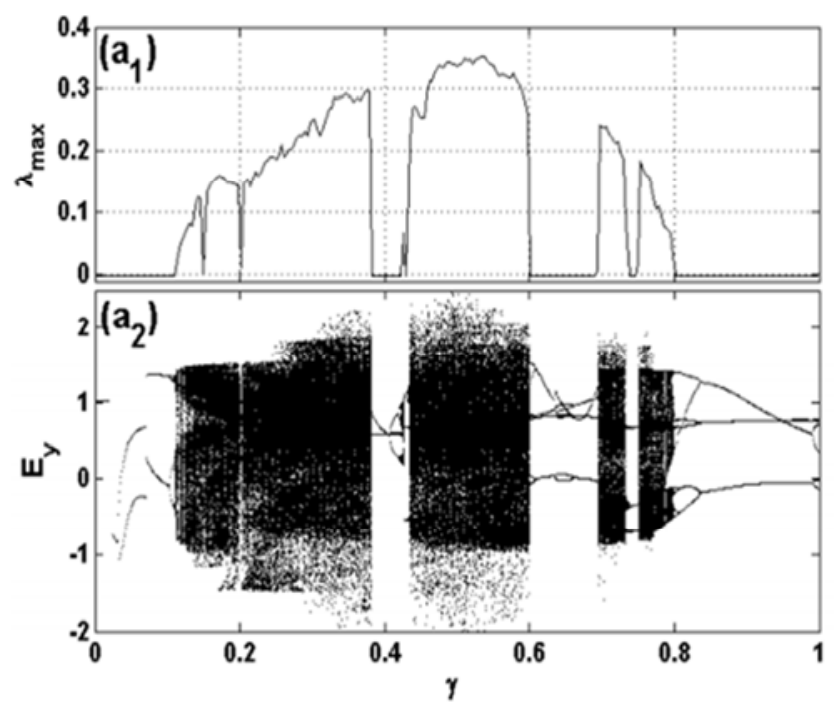
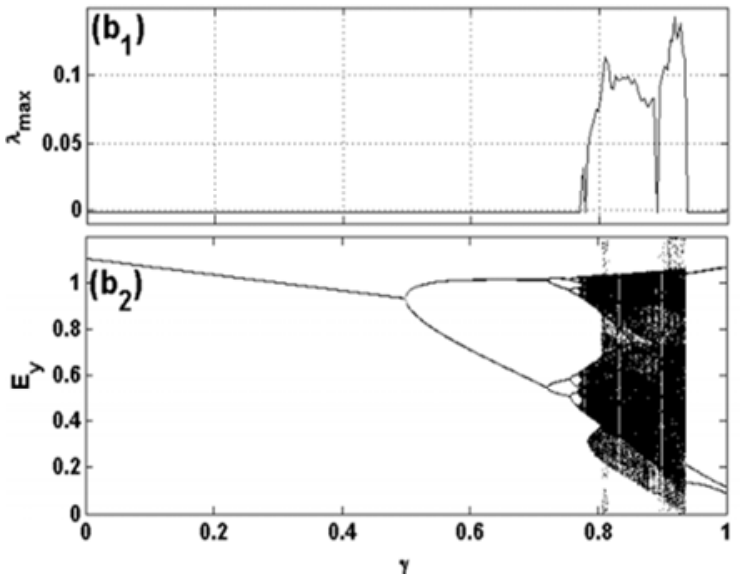

Figure 3. Bifurcation diagrams (for three distinct values of external amplitude) showing local maxima of the imaginary part of the complex electric field $E_{y}$ and corresponding graph of Lyapunov exponents versus injected field strength $\gamma$. (a) for $F_{0}=0$ (without external excitation), (b) for $F_{0}=3$.
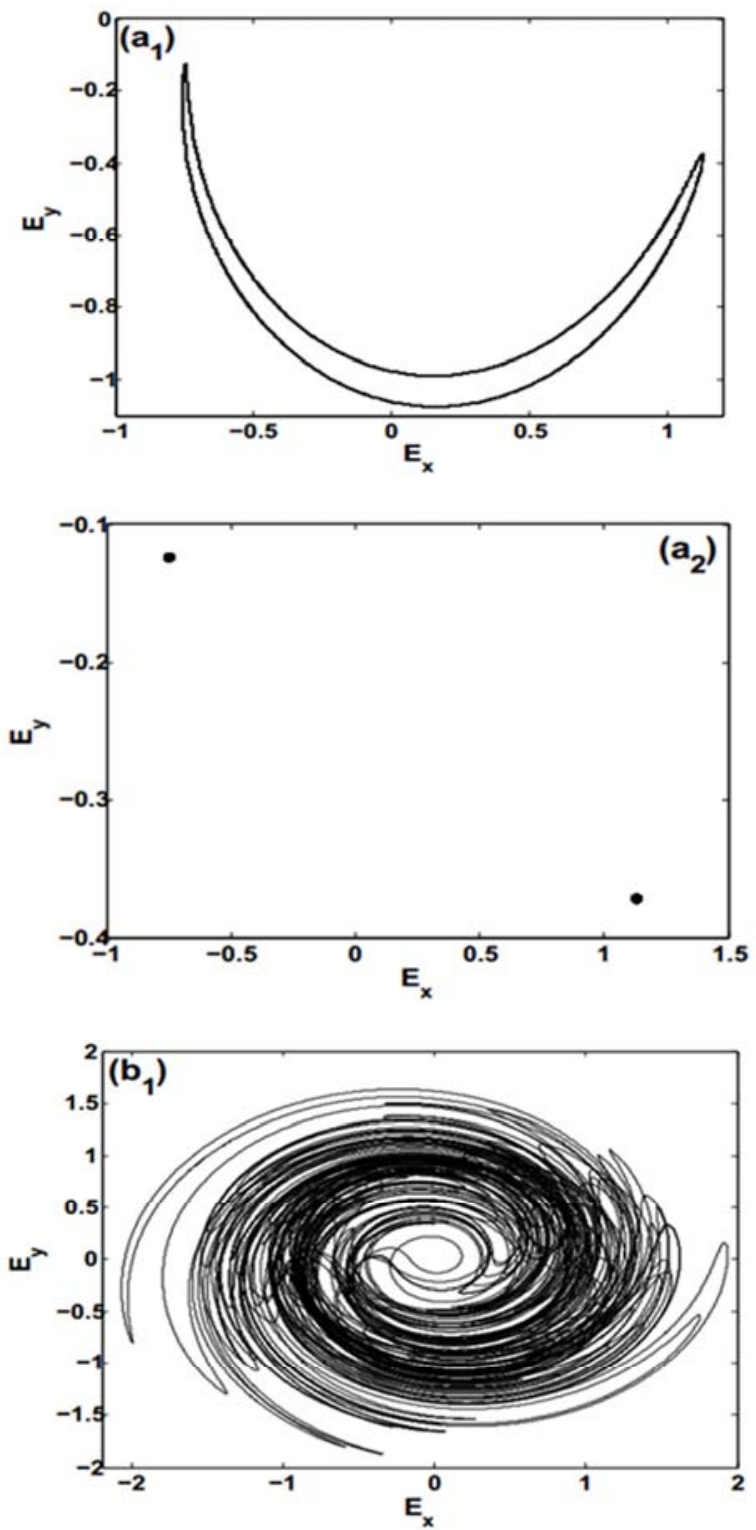

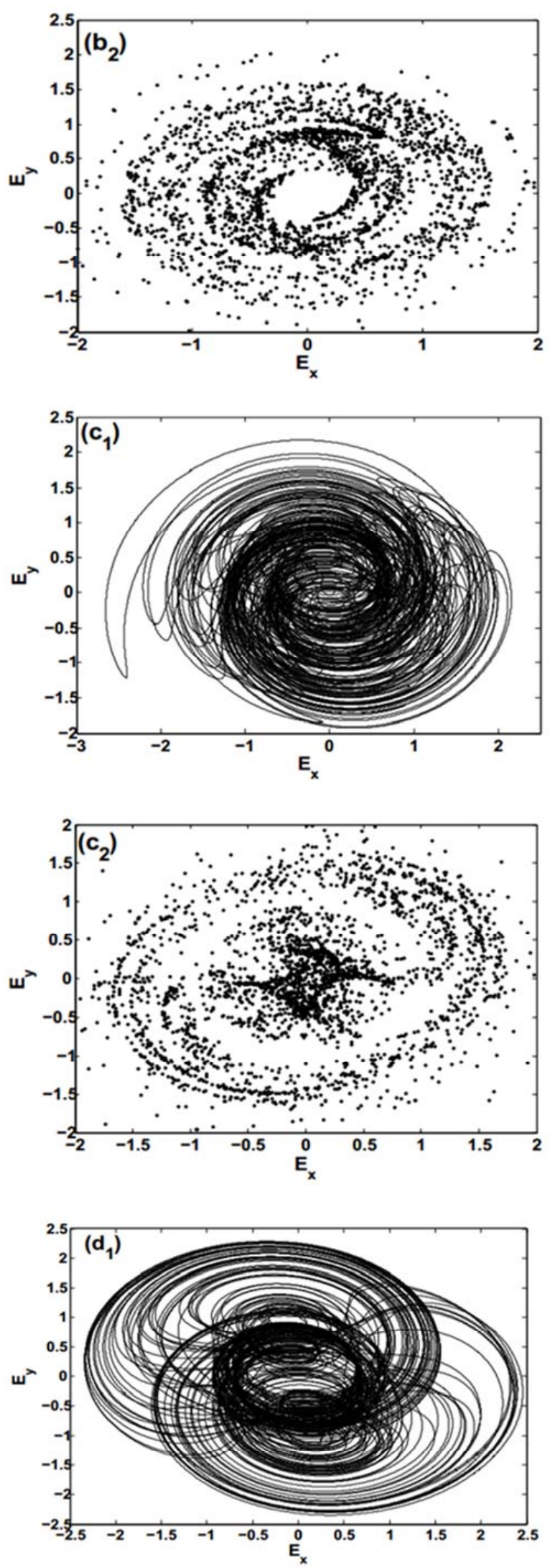
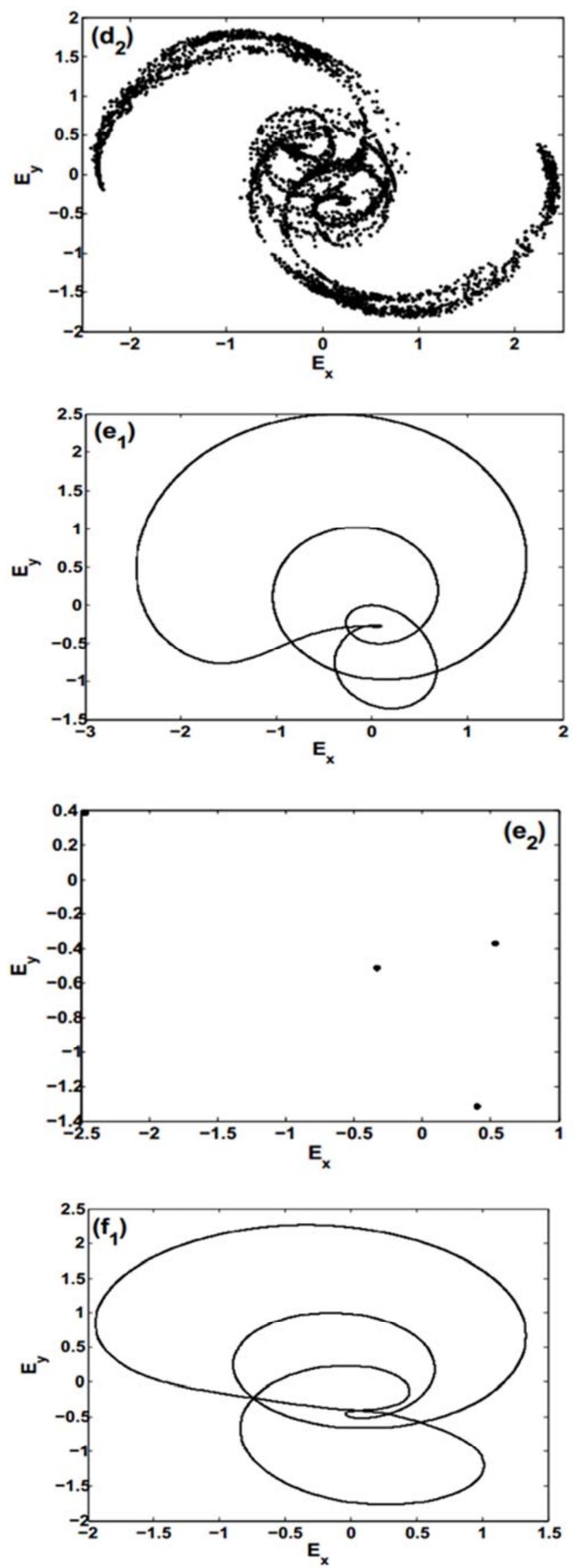

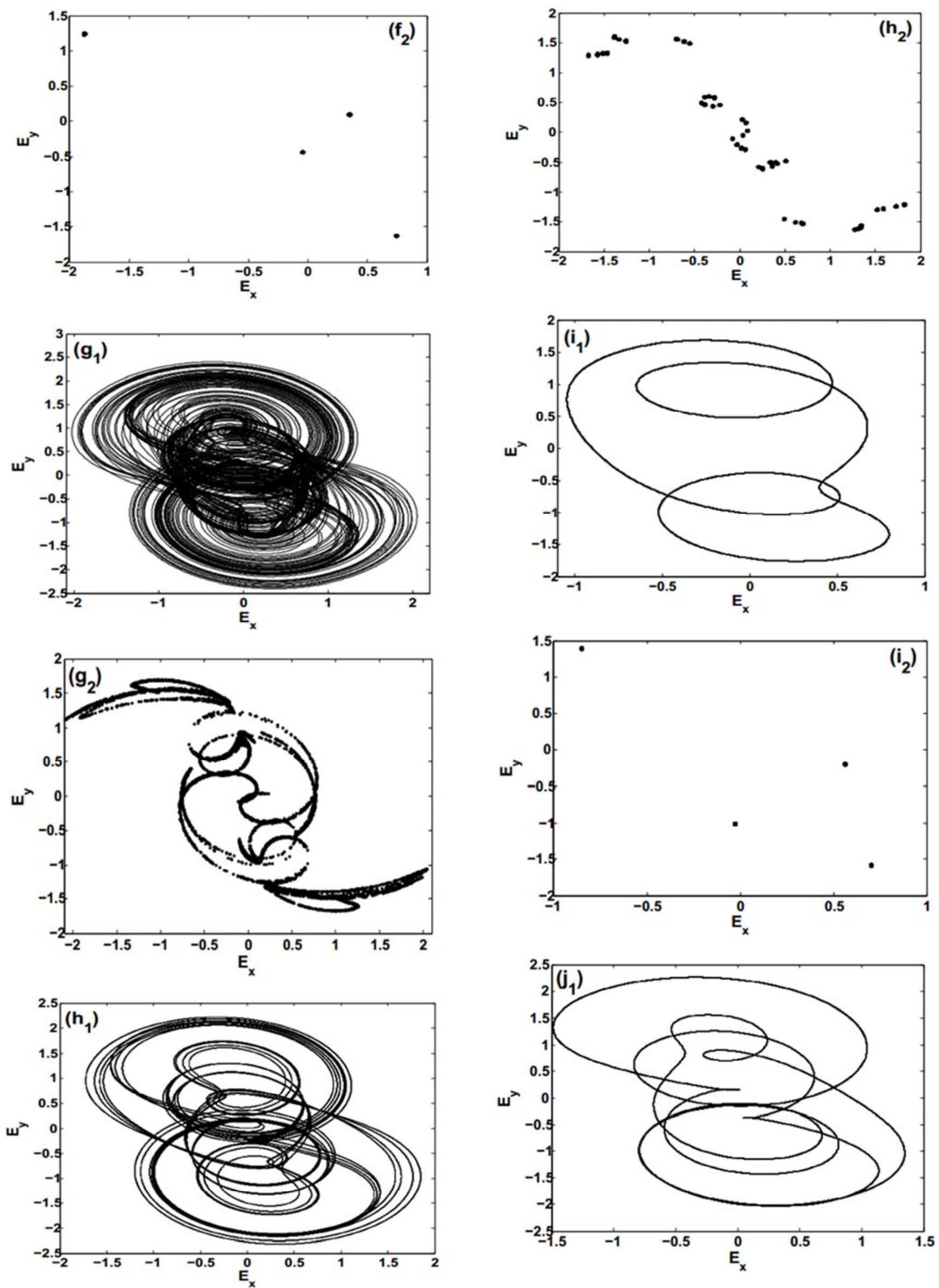


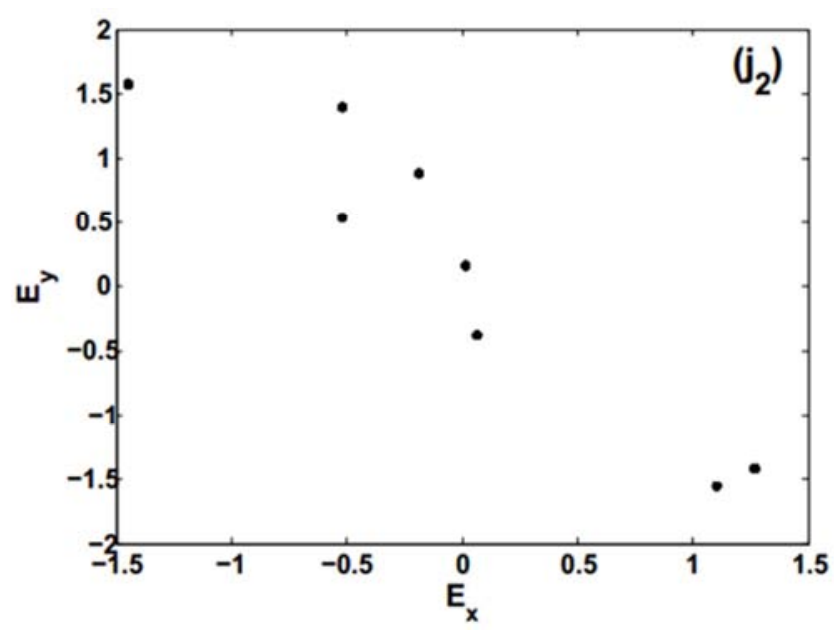

Figure 4. Numerical phase portraits and corresponding Poincaré sections in the perturbed diode laser for discrete values of the external excitation $F_{0}$ : (a) asymetric period-1 for $F_{0}=0.1$, (b) asymetric chaotic attractor for $F_{0}=0.3$ (c) asymetric chaotic attractor for $F_{0}=1$, (d) symetric chaotic attractor for $F_{0}=2.5$, (e) asymetric period-1 for $F_{0}=3$, (f) asymetric period- 1 for $F_{0}=3.2$, (g) symetric chaotic attractor for $F_{0}=4$, (h) symetric torus for $F_{0}=4.4$, (i) asymetric period-1 for $F_{0}=4.6$ and (j) asymetric period-1 for $F_{0}=5$. The initial conditions are chosen as: $\left(E_{x} ; E_{y} ; n\right)=(-0.09 ; 0.09 ;-0.9)$.

\subsection{Two Parameters Diagrams, Coexisting of Attractors}

In the previous subsection, we investigated the effect of external amplitude and excitation frequency on the dynamic laser. In that subsection, we fixed excitation frequency at $7000 \mathrm{~Hz}$ and chosen discrete values of external amplitude in order to explore its dynamics. In this section, we fix fourth distinct values of external frequency and investigate the dynamical behaviors of the laser model when external amplitude and laser pump are varied simultaneously. The investigation is realized through two parameter diagrams when amplitude and pump varying between 0.2 and 2 (see Figure 5). In Figure 5, each color is attached to a specific dynamical behavior. For example, blue colors are associated to absence of oscillation $(\lambda<0)$, red and yellow colors to chaotic motion $(\lambda>0)$, and cyan colors to regular motion $(\lambda=0)$. One can notice that, when external frequency fixed at $1000 \mathrm{~Hz}$ (resp $9000 \mathrm{~Hz}$ ), the dynamical behavior of laser tends to be regular (resp no chaotic) (see Figure 5a and 5b).

When the external frequency is fixed $5000 \mathrm{~Hz}$, the dynamic of the laser tends to be chaotic (see Figure 5b).

However, for the external frequency fixed at $7000 \mathrm{~Hz}$, we observe the occurrence of regular and chaotic attractors. When external frequency and external amplitude are fixed to $7000 \mathrm{~Hz}$ and 10 respectively, we plotted diagram of Figure $6 \mathrm{~b}$ by increasing respectively decreasing the injected field strength in the region $0.6 \leq \gamma \leq 0.66$ with initial conditions $\left(E_{x}(0) ; E_{y}(0) ; n(0)\right)=(0 ;-1 ;-0.1)$. One can notice between 0.6418 and 0.6488 the coexistence of chaotic and periodic attractors (see Figure 6b). By fixing the discrete value of the pump at 0.6448 in the coexisting region, we plotted portraits of Figure $6 \mathrm{c}, 6 \mathrm{~d}$ and $6 \mathrm{e}$. Figure $6 \mathrm{e}$ shows the cross section of initial conditions leading to each coexisting attractors of Figure 6c. Numerical analysis of Eq (2) for $\omega=$ $0.13, \alpha=6.6, \gamma=0.46, f=7000 \mathrm{H}_{\mathrm{z}}, \mathrm{F}_{0}=0.009, \Gamma=0.0973$ and $\mathrm{B}=0.0395$ reveal existence of metastable chaos. Figure 7 shows the time-domain waveform (left panel) and phase portraits (right panel) of the chaotic attractor with transient chaos. We have plotted in Figure $7 \mathrm{a}$ and $7 \mathrm{~b}$ the time traces $E_{y}(\tau)$ and the phase portrait of state $\left(E_{x}-E_{y}\right)$ for $\left(E_{x}(0) ; E_{y}(0) ; n(0)\right)=(-0.09 ; 0.09 ;-0.9)$. Red and black colors are associated respectively to the torus and chaotic orbits.

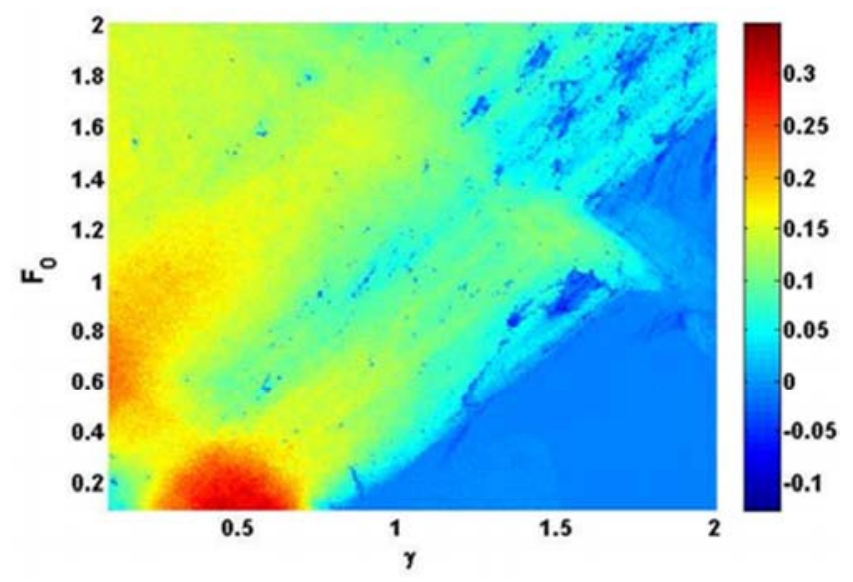

(a)

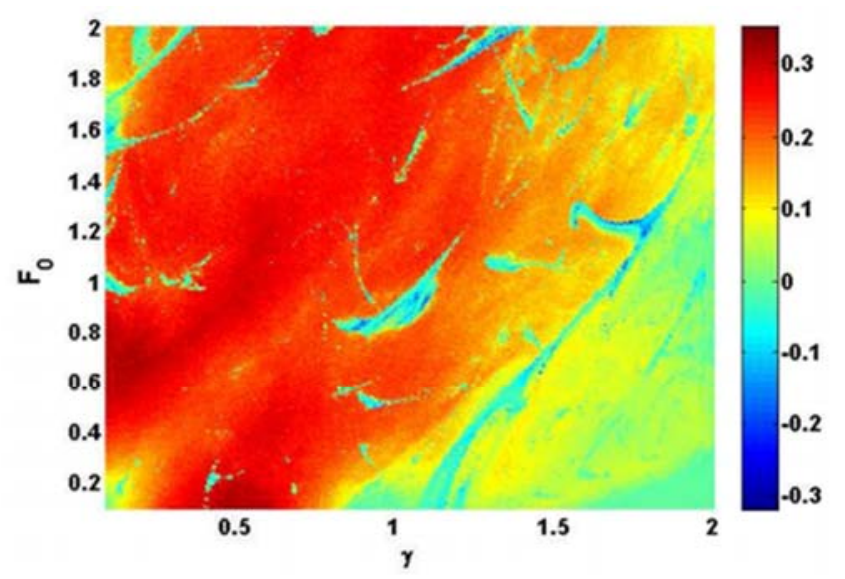

(b)

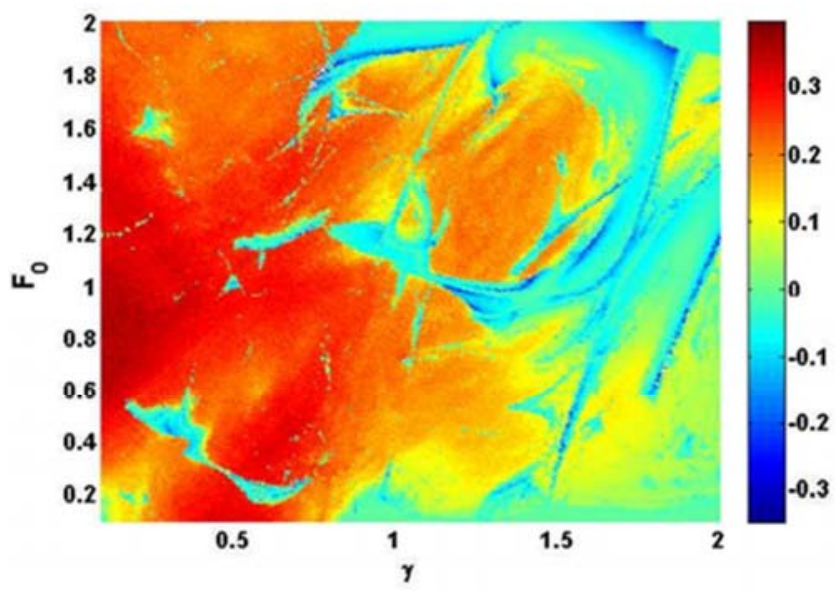

(c) 


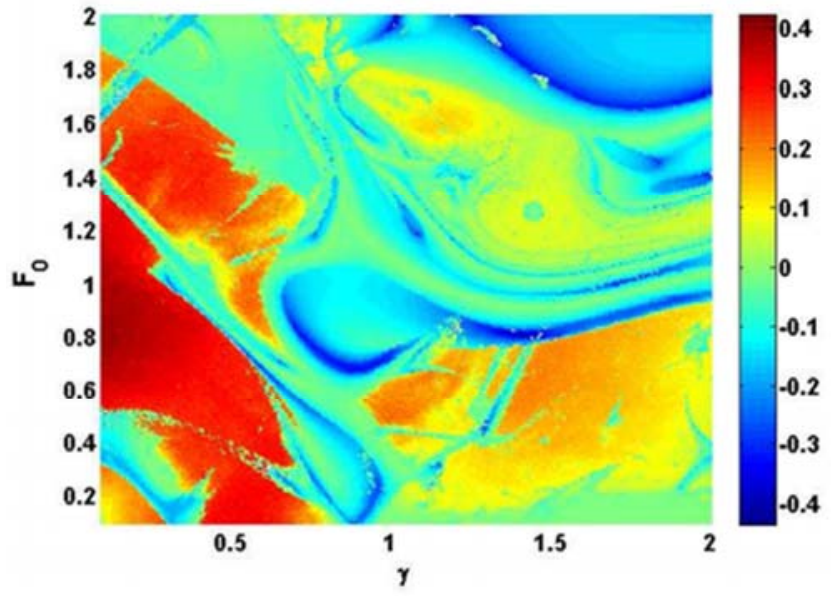

(d)

Figure 5. Two parameters diagrams in the $\left(\gamma, F_{0}\right)$ space showing the global dynamics of the perturbed diode laser based on the sign of the largest Lyapunov exponents. Chaotic regions are painted in red and yellow colors while regions of regular oscillations are associated to cyan color. Diagrams of Figure 5 are obtained for fourth discrete values of external excitation: (a) for external frequency $f=1000 H_{z}$, (b) for $f=5000 H_{z}$, (c) for $f=7000 H_{z}$ and (d) for $f=9000 H_{z}$.
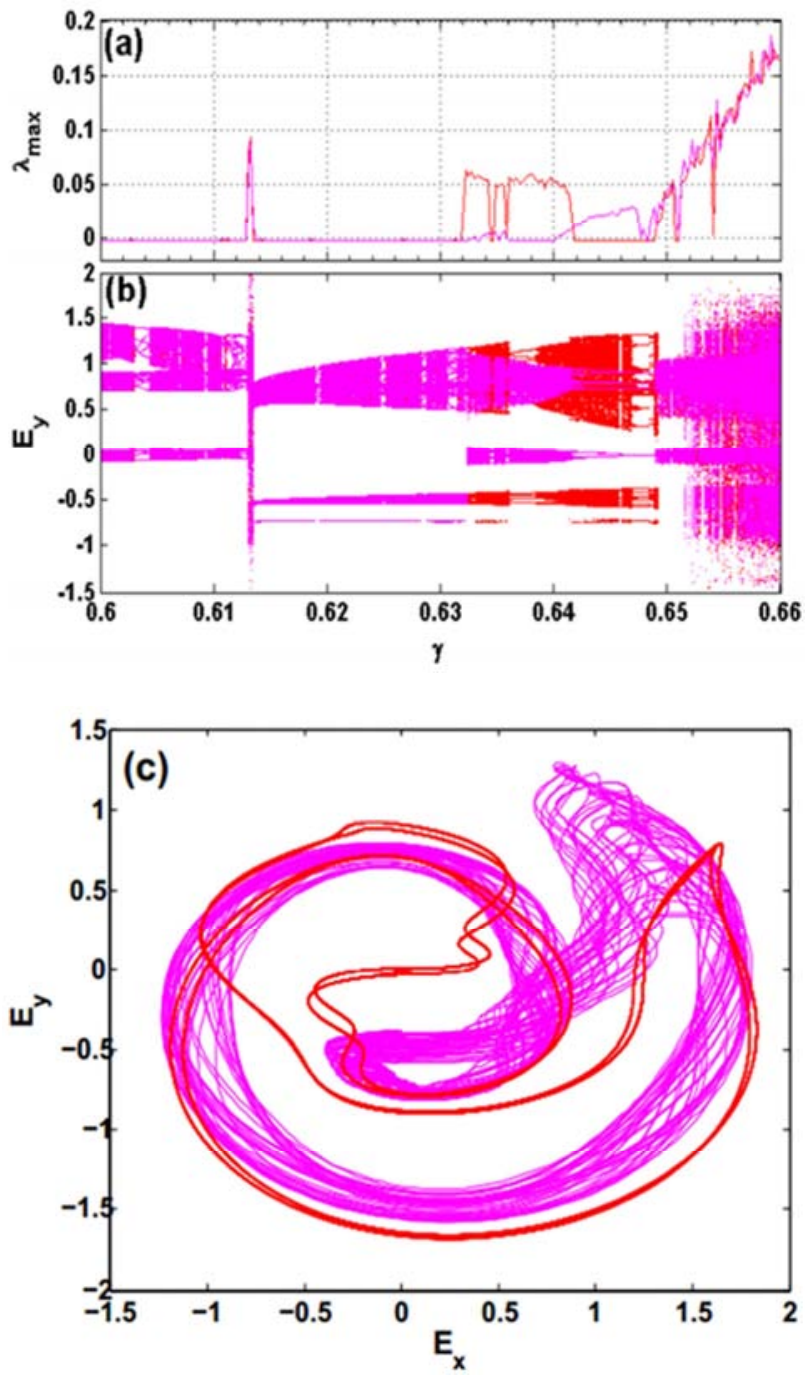
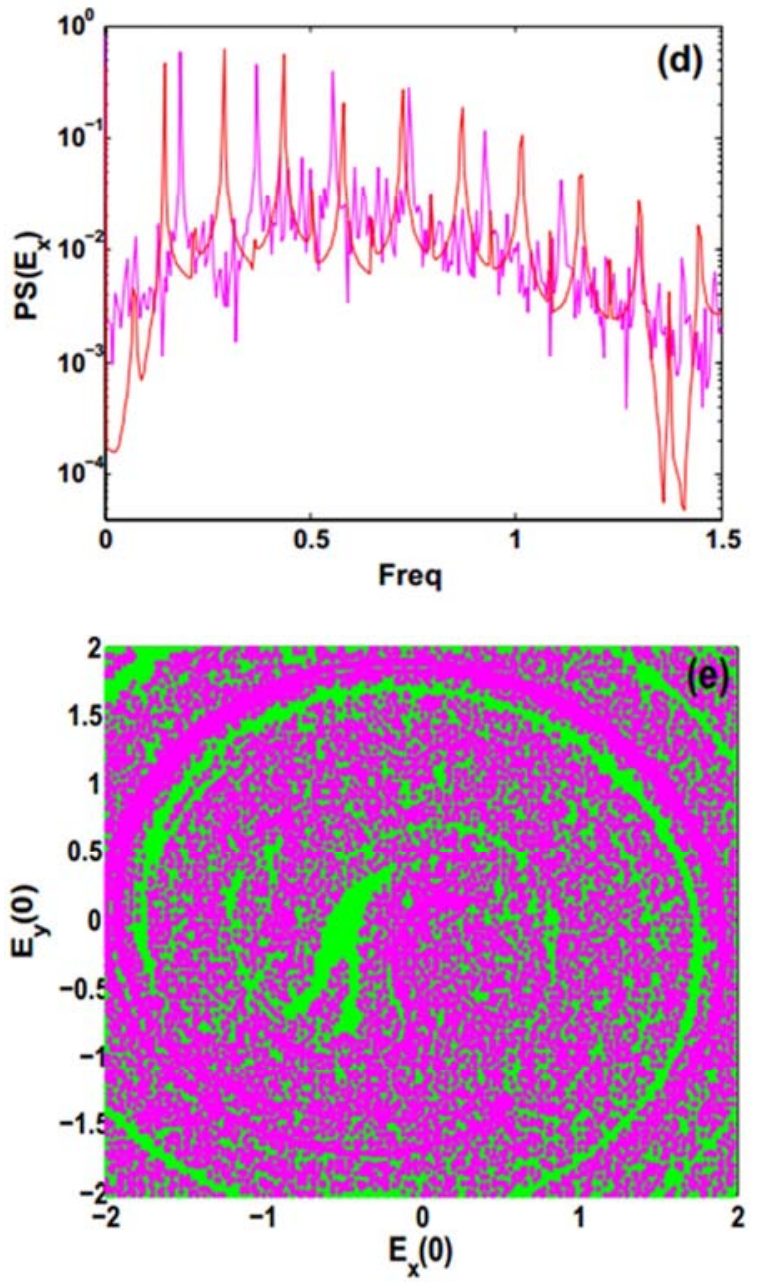

Figure 6. (a) Bifurcation diagrams showing local maxima of the imaginary part of the complex electric field $E_{y}$ and corresponding graph of Lyapunov exponents (b) versus injected field strength $\gamma$. Superimposed red and magenta diagrams correspond respectively to increased and decreased values of injected field strength. Phase portrait (c) showing coexistence of two attractors (period and chaotic) with their corresponding frequency spectra (d) for $\gamma=0.6448$ and $F_{0}=10$; initial condition are $\left(E_{x} ; E_{y} ; n\right)=$ $(0 ;-0.09 ;-0.09)$ for period-2 limit cycle (red) and $(0 ; 0.9 ;-0.09)$ for the chaotic one (magenta). Cross section basin of initial condition in the plane leading to each coexisting attractors is depicted in (e), where magenta and green colors are associated respectively to periodic and chaotic attractors. The other initial condition was fixed as: $n(0)=-0.1$ while $-2 \leq E_{x}(0) \leq 2$ and $-2 \leq E_{y}(0) \leq 2$.

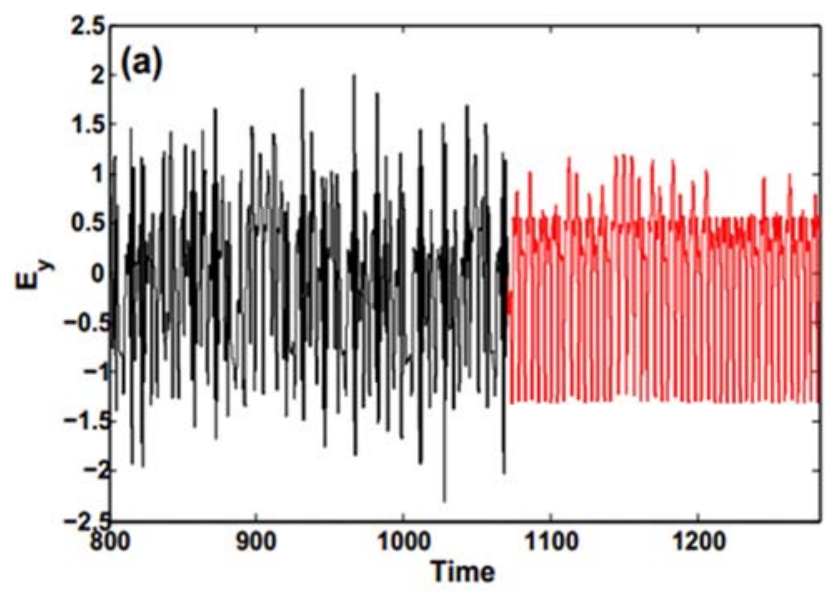




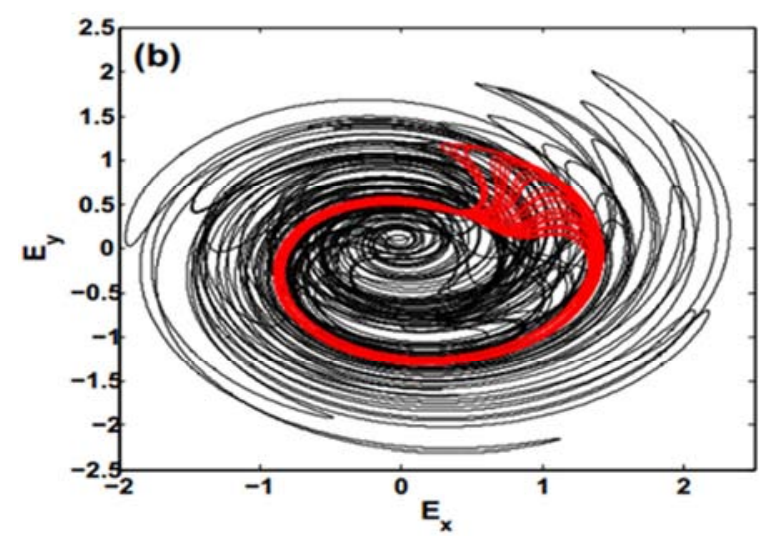

Figure 7. Transition dynamics from transient chaotic to torus behavior global time-domain waveform of the variable $E_{y}$ in the time interval [800, 1300]. Initial conditions: $\left(E_{x} ; E_{y} ; n\right)=(-0.09 ; 0.09 ;-0.9)$.

\subsection{Pspice Simulations}

In this section, we are verifying through PSpice environment the dynamical behavior of laser model as predicted by the theoretical results. Indeed, PSpice has been widely used in several paper to confirm numerical investigations [24]. One of the main advantages of PSpice is the possibility to vary initial conditions of components/reservoirs. PSpice results allow one to validate numerical results like multistability [24] (i.e., coexistence of attractors). Also, PSpice is a suitable environment in which the design and experimentation of electronic circuit help to choose the proper parameters for numerical simulation. Henceforth, we have realized the corresponding analog simulator of laser model as presented in Figure 8. The analog electronic circuit is obtained using operational amplifiers, analog multipliers, resistors, capacitors and DC bias voltages. Active components are polarized with a $\mp 15$ DC bias voltage source. Applying Kirchhoff's circuit laws to Figure 8, we have derived the following state equations:

$$
\begin{gathered}
R_{1} C_{1} \dot{v}_{x}=\frac{R_{4}}{R_{7}} E_{1}+\frac{R_{4}}{10 R_{8}} v_{x} v_{z}-\frac{R_{4}}{10 R_{9}} v_{y} v_{z}+\frac{R_{4}}{R_{w}} v_{y} \\
\quad-\frac{R_{01}}{R_{0}} \sin (2 \pi f t) \\
R_{2} C_{2} \dot{v}_{y}=\frac{R_{5}}{10 R_{11}} v_{x} v_{z}-\frac{R_{5}}{10 R_{10}} v_{y} v_{z}-\frac{R_{5}}{R_{w}} v_{x} \\
R_{3} C_{3} \dot{v}_{z}=\frac{R_{6}}{R_{18}} E_{2}-\frac{R_{6}}{10 R_{13}} v_{y}^{2}-\frac{R_{6}}{10 R_{12}} v_{x}^{2}-\frac{R_{6}}{100 R_{16}} v_{z} v_{y}^{2} \\
-\frac{R_{6}}{100 R_{15}} v_{z} v_{x}^{2}-\frac{R_{6}}{R_{17}} v_{z}+\frac{R_{6}}{R_{14}} v_{z}
\end{gathered}
$$

By fixing $C_{1}=C_{2}=C_{3}=C, R_{4}=R_{5}=R_{6}=R \quad$ and considering the time constant of the three integrators as $R C$, the parameters of the laser model can be expressed in terms of the circuit parameters as follows: $\gamma=R E_{1} / R_{7}$, $\alpha=R / 5 R_{11}=R / 5 R_{9}, \omega=R / R_{\omega}, 2 B=$

$R / 100 R_{15}=R / 100 R_{16}, 2 \Gamma=R / R_{17}$ and $F_{0}=R_{01} / R_{0}$. For the typical parameters $\omega=0.13, \alpha=6.6, \gamma=0.04, \Gamma$ $=0.0973$ and $\mathrm{B}=0.0395$ the corresponding circuit parameters can be taken as follows: $R_{9}=R_{11}=0.1515 k \Omega, R_{7}=$
$125 \mathrm{k} \Omega, R_{\omega}=38.46 \mathrm{k} \Omega \quad, \quad R_{15}=R_{16}=0.632 \mathrm{k} \Omega \quad$ and $R_{17}=25.69 \mathrm{k} \Omega$. The capacitor values are: $C_{1}=C_{2}=C_{3}=100$ $\mathrm{nF}$. We provide in Figure 9 sample Pspice results and their numerical corresponding for the parameter range above. One can notice very good qualitative agreement between theoretical and Pspice results.

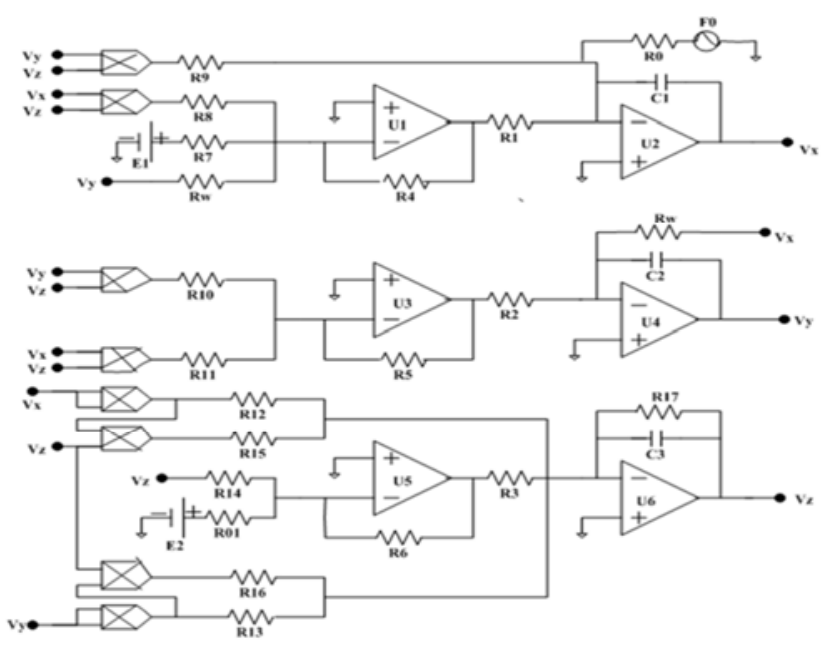

Figure 8. Circuit emulator of the laser model designed in Pspice environment.
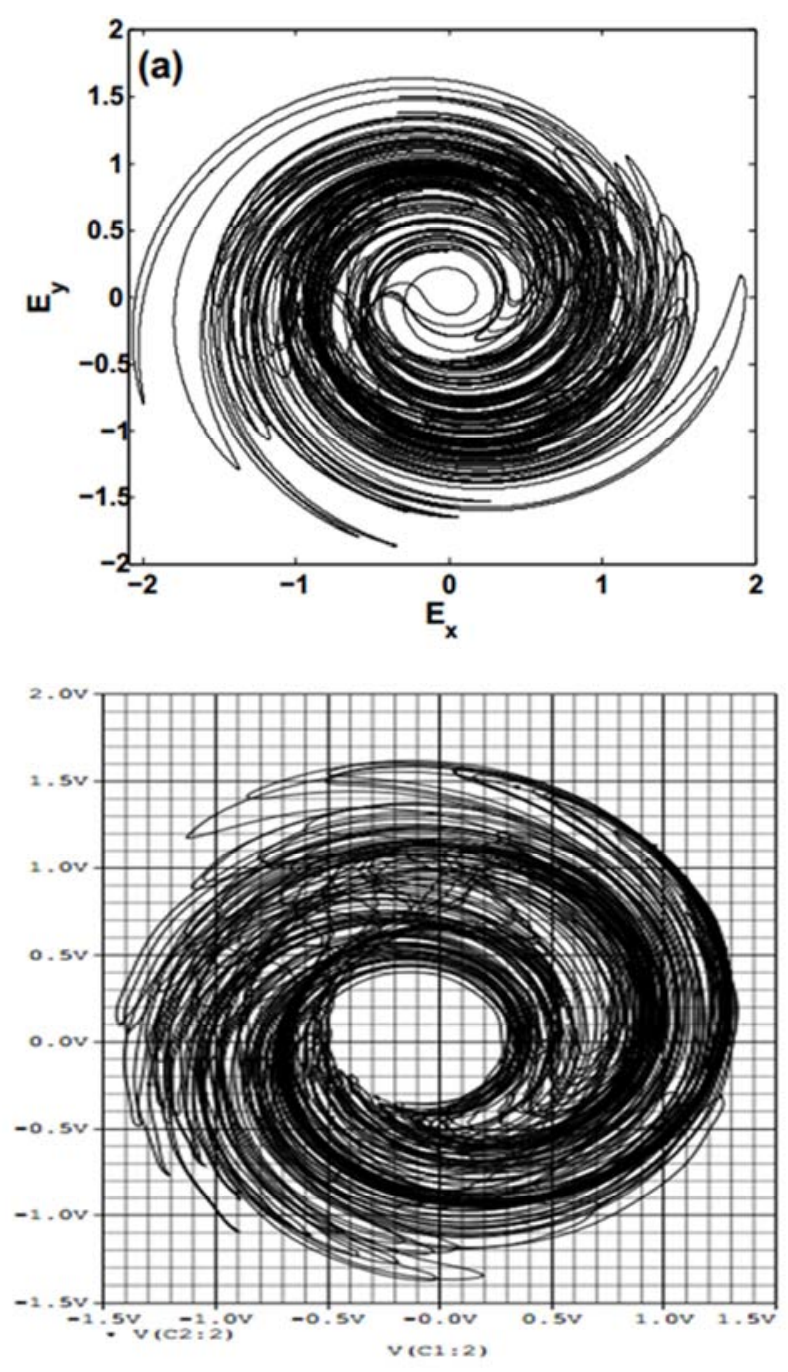

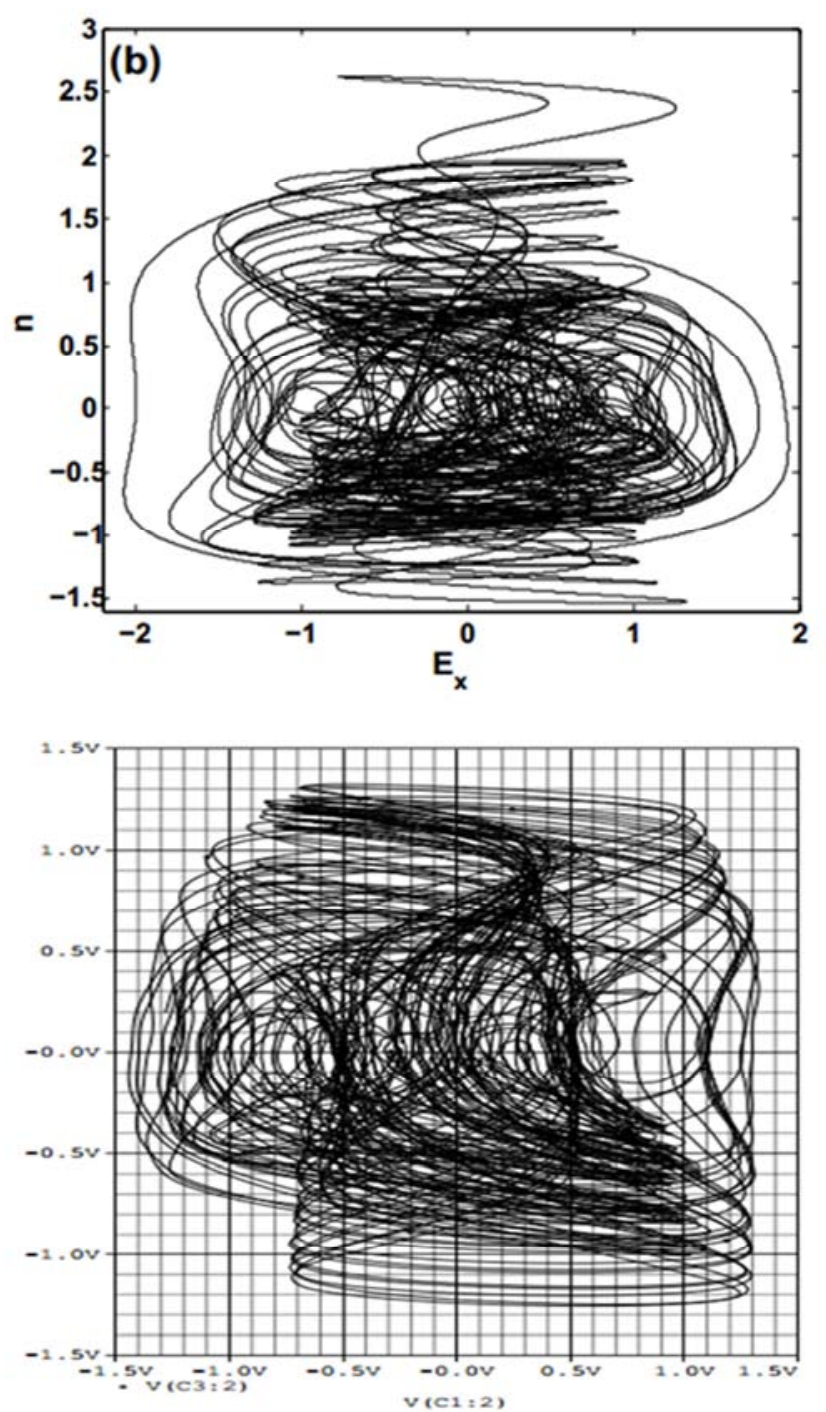

Figure 9. The phase portraits and corresponding Pspice obtained for $R_{c}=2500 \mathrm{k} \Omega, R_{b}=0.166 \mathrm{k} \Omega, R_{9}=R_{11}=0.1515 \mathrm{k} \Omega, R_{7}=125 \mathrm{k} \Omega, R_{\omega}=$ $38.46 \mathrm{k} \Omega, R_{15}=R_{16}=0.632 \mathrm{k} \Omega, R_{17}=25.69 \mathrm{k} \Omega$ and $F_{0}=0.3 \mathrm{~V}$. With initial conditions $\left(V_{c 1}(0), V_{c 2}(0), V_{c 3}(0)\right)=(0 \mathrm{~V}, 0 \mathrm{~V},-0.1 \mathrm{~V})$.

\section{Conclusion}

In this paper, a rate equation model of semiconductor laser with periodic perturbation has been considered and investigated. Using nonlinear analysis techniques such as bifurcations diagrams, Lyapunov exponents, phase portraits, Poincaré sections, basin of attraction and two parameters diagram, the dynamics of the model has been characterized. Several signals including periodic, chaotic and torus are observed (bifurcation diagrams) in the injected laser (slave) for some discrete values of amplitude of external perturbation. The occurrence of ten attractors including three symmetric and six asymmetric attractors are underlined. Moreover, the study of the dynamics is done through the two parameters diagrams by varying simultaneously external amplitude and injected field strength. This study reveals a large periodic region (resp a large chaotic region) for external frequency fixed at $1000 \mathrm{~Hz}$ (resp at $5000 \mathrm{~Hz}$ ). Cross section basin of initials conditions showing the domain of initials states leading to each coexisting attractor's is provided (ie coexistence of periodic and chaotic attractors). The graphs of phase portraits were plotted to confirm transient dynamics from transient chaos to torus behaviors. Finally, Pspice simulations are performed to support numerical results.

\section{References}

[1] M. S. Torre, C. Masoller «Exploiting the nonlinear dynamics of optically injected semiconductor lasers for optical sensing» Photonics, vol. 2, 7pp., 2019. J. Clerk Maxwell, A Treatise on Electricity and Magnetism, 3rd ed., vol. 2. Oxford: Clarendon, 1892, pp. 68-73.

[2] B. Bao, H. Qian, J. Wang, Q. Xu, M. Chen, H. Wu and Y. Yu «Numerical analyses and experimental validations of coexisting multiple attractors in Hopfield neural network » Nonlinear Dyn, vol. 3, 2017. K. Elissa, "Title of paper if known," unpublished.

[3] G. Cai, P. Hu and Y. Li «Modified function lag projective synchronization of a financial hyperchaotic system » Nonlinear Dyn, vol. 69, 2012.

[4] W. Horton, R. S. Weigel and J. C. Sprott «Chaos and the limits of predictability for the solar-wind-driven magnetosphere-ioosphere system »Phys. Plasma, vol. 6, pp. 2946-2952, 2001.

[5] Ayub khan sanjay kum «Mesure of chaos and adaptive synchronization of chaotic satellite systems» Int J of Dyn and Control., vol. 4, pp. 283-294, 2002.

[6] S. Wieczorek, B. Krauskopf and D. Lenstra, «A Unifying View of Bifurcations in Semiconductor Laser Subject to Optical Injection» Opt Comm, vol. 172, pp. 279-295, 1999.

[7] K. Murali and M. Lakshmanan «Bifurcation chaos of the sinusoidally-driven chua's circuit» Int. J. of Bif and chaos, vol. $1, \mathrm{n}^{\circ} 2$, pp. 369-384, 1991.

[8] C. Amanda and P. C. Rech «Changes in the dynamics of a Rossler oscillator by an external forcing» Chinese Phys. Lett, vol. 30, 5pp, 2013.

[9] Q. Xu, Z. Song, H. Bao, M. Chen and B. Bao «Two-neuron -based non-autonomous memristive Hopfield neural network: Numerical analyses and Hardware experiments» Int. J. Electron. Commun, vol. 96, pp. 66-74, 2018.

[10] A. Ngo Mouelas, T. Fonzin Fozin, R. Kengne, J. Kengne, H. B. Fotsin and B. Z. Essimbi «Extremely rich dynamical behaviors in a simple nonautonomous Jerk system with generalized nonlinearity: hyperchaos, intermittency, offset-boosting and multistability» Int. J. of Dyn and Control, 10, 2019.

[11] C. Harder, J. Katz, S. Margalit, J. Shacham and A. Yariv «Noise equivalent circuit of semiconductor laser diode» IEEE. J. of Quantum Elect, vol. 18, n 3, 5 pp, 1982.

[12] K. Hwang, J. B. Gao and J. M. Liu «Noise-induced chaos in an optically injected semiconductor laser model» Phys. Rev E, vol. 61, 9 pp, 1999.

[13] B. Garbin, A. Dolcemascolo, F. Prati, J. Javaloyes, G. Jissoni and S. Barland «Refractory period of an excitable semiconductor laser with optical injection» Phys. Rev E, vol. 95, 8 pp, 2017. 
[14] F. Gustave, L. Columbo, G. Tissoni, M. Brambilla, F. Prati and S. Barland «Phase solitons and domain dynamics in an optically injected semiconductor laser» Phys. Rev A, vol. 93, $10 \mathrm{pp}, 2016$.

[15] A. Pimenov, E. A. Viktorov, S. P. Hegarty, T. Habruseva, G. Huyet, D. Rachinskii and A. G. Vladimirov «Bistability and hysteresis in an optically injected two-section semiconductor laser» Phys. Rev E, vol. 89, 7 pp, 2014.

[16] M. S. Pramod, T. Yang, K. Pandey, M. Giudici and D. Wilkowski «Selective injection locking of a multi-mode semiconductor laser to a multi-frequency reference beam» Eur. Phys. J. D, vol. 68, 5 pp, 2014.

[17] S. Wieczorek, B. Krauskopf, T. B. Simpson and D. Lenstra, "The Dynamical Complexity of Optically Injected Semiconductor Lasers," IEEE Physics Reports, vol. 416, pp. 1-128, 2005.

[18] S. Lang, "Injection locking properties of a semiconductor laser" IEEE J. Quantum electron, Vol. 18, 1982.
[19] S. Kobayashi, T. Kimura "Injection locking characteristics of an AlGaAs semiconductor laser" IEEE J. Quantum electron, Vol. 16, 1980.

[20] F. Mogensen, H. Olesen, G. Jacobsen "FM noise suppression and linewidth reduction in an injection-locked semiconductor laser"Electron. Len, Vol. 21, 1985.

[21] J. Kengne, S. Jafari, Z. T. Njitacke, M. Yousefi Azar Khanian, A. Cheukem «Dynamic analysis and electronic circuit implementation of a novel 3D autonomous system without linear terms» Commun Nonlinear Sci Numer Simulat, vol. 52, pp. 62-76, 2017.

[22] Z. T. Njitacke, J. Kengne, A. Nguomkam Negou «Dynamical analysis and electronic circuit realization of an equilibrium free 3D chaotic system with a large number of coexisting attractors» Optik, vol. 130, pp. 356-364, 2017. 